Proceedings of the International School and Conference on Optics and Optical Materials, ISCOM07, Belgrade, Serbia, September 3-7, 2007

\title{
Models of Interactions of Laser Beams with Materials of Interest for Optical Components and Provoked Damages
}

\author{
M. SrećKovića ${ }^{a}$,J. Ilić ${ }^{b}$, A. KovaČeviĆc,*, S. PANtelić ${ }^{a}$, \\ Z. Latinovićd ${ }^{\prime}$ N. Borna ${ }^{e}$ And V. ĆOSOVIĆ ${ }^{e}$ \\ ${ }^{a}$ Faculty of Electrical Engineering, University of Belgrade, Serbia \\ ${ }^{b}$ Faculty of Mechanical Engineering, University of Belgrade, Serbia \\ ${ }^{c}$ Institute of Physics, Pregrevica 118, 11080 Belgrade, Serbia \\ ${ }^{d}$ IRADIA, Sremska Kamenica, Serbia \\ ${ }^{e}$ Faculty of Technology and Metallurgy, University of Belgrade, Serbia \\ Models of interactions of laser beams with materials of interest for op- \\ tical components are presented in this paper. Special attention is paid to \\ damages which appear within both active materials and optical components \\ along the path of a propagating beam with theoretical treatment via various \\ models.
}

PACS numbers: $81.40 . \mathrm{Wx}, 61.80 . \mathrm{Ba}, 42.62 .-\mathrm{b}$

\section{Introduction}

Among various material modifications (labeled as reversible, irreversible, temporary, permanent, unexpected/"unwanted"/"negative" or not, etc.), damaging attracts much attention nowadays. The investigation of the cases on the border between temporary and permanent change provides the information on the resistivity of optical components [1-11].

Much research has been done in material exposition to laser beams of various dynamical operating regimes [1-11]. There are various data considering the threshold of damages, namely, laser-induced damage threshold (LIDT) [1-3], showing the tendency for standardization of fracture-appearing phenomena. There are various established procedures for determining the damage threshold. In studying damages induced via dielectric, thermal, Brillouin or other breakdown (including mechanical) processes, the material purity plays an important role. For instance, if inclusions in optical components are in the form of metal particles, specific cracks appear and damages provoked in active materials (ruby, glass) are explained by

*corresponding author; e-mail: Aleksander.Kovacevic@phy.bg.ac.yu 
specific approaches $[1-3,10]$. Besides various theoretical approaches, with plasma phenomena included or not, experimental results are the most reliable confirmation of recommended model. The tendency to replace glass with plastic material theoretically requires inclusion of various new models for damage explanation.

Various optical materials and elements (lenses and other glass or polymer components) have been exposed to laser pulses and their damages have been analyzed by microscopic and other methods. The threshold (LIDT) depends on the state of the surface of an optical component prior to laser interaction. The analysis includes: the influence of the internal electric field (the field depends on the shape of micro-scratches) and the correction of the refractive index by the Sellmeier approximations for less known materials.

It is necessary to record the changes of the material characteristics during its operating life. According to the type of both optical components and lasing media, the following cases are considered: (a) sample degradation; (b) the tasks which prolong the component life; (c) possible technological reparative processes. Laser irradiation near (below) damage threshold might improve mechanical performances and properties of some fibers or detectors.

High thermal gradients commonly arise in active materials during their operation. The calculation of thermal stresses provoked in the materials is complex, but allows the analysis of lasing processes in some (chosen) configurations. During the operation, stresses which exceed the limits appear in the materials with various impurities included, which frequently lead to mechanical cracks. The detection of these states, and methods for their analysis, are considered through contemporary methods of mechanical stress control and compared with theoretical considerations of crack processes in previously mentioned cases (inclusions of metallic particles) [10].

In the field of quantum electronics, some materials may have multiple roles. They are used as active materials, but they are also used as windows or other necessary optical elements. For instance, GaAs is used as a window for some types of gas lasers, and it can also be an active material. The question of laser output power limits, amplification degrees and throughput fluxes arises.

In high power lasers, multistage amplification is unavoidable. In technical calculations, expressions that involve lasing thresholds and differential efficiency are derived [4]. Factors causing the discrepancies are connected with: the influence of non-homogeneity which leads to the appearance of saturation, the influence of mode structure and the influence of overheating. High thermal gradients lead to damages showing permanent deterioration of laser parameters as well as burning and "shake" of crystals. Laser efficiency, thermal and lasing thresholds define maximum operating temperatures [4].

\section{Experimental techniques}

Many types of materials are frequently used in experiments with short $\left(\mathrm{LiNbO}_{3}, \mathrm{LaTaO}_{3}, \mathrm{Na}_{2} \mathrm{NbO}_{3} \ldots\right)$ and both short and long (organic glasses, 
poly(methyl methacrylate) (PMMA) and polystyrene [6]) laser pulses. In all materials, surface and volume processes are commonly monitored. Fused silica, BK, TF, K8, GLS-1, GLS-2 glasses, as well as linear crystals (KDP, $\mathrm{LiTiO}_{3}$, ruby), are worth mentioning as interesting materials for laser processing.

Micro-fractures have been monitored and analyzed. Three groups of specimens have been examined: (1) alphafilcon specimens - co-polymer of 2hydroxethyl methacrylate, N-vinyl pyrolidone and 4-tertiary butyl-2-hydroxycyclohexyl methacrylate, which contains $66 \%$ of water when immersed in appropriate sterile $\mathrm{NaCl}$ and borate buffer solution (density of $1.075 \mathrm{~g} / \mathrm{cm}^{3}$, refractive index of $n=1.390$, oxygen permeability of $32 \times 10^{-11}\left(\mathrm{~cm}^{3} \mathrm{O}_{2} \cdot \mathrm{cm}\right) /\left(\mathrm{cm}^{2} \cdot \mathrm{s} \cdot \mathrm{mmHg}\right)$ at $35^{\circ} \mathrm{C}$ [5]); (2) itabisfluorofocon polymer specimens [5] — methyl-methacrylate-fluoritaconate-siloxanyl co-polymers which contains $1 \%$ of water when immersed in mentioned solution (density of $1.27 \mathrm{~g} / \mathrm{cm}^{3}$, refractive index of $n=1.435$, oxygen permeability of $45^{*}\left(24^{* *}\right) \times 10^{-11}\left(\mathrm{~cm}^{3} \mathrm{O}_{2} \cdot \mathrm{cm}\right) /\left(\mathrm{cm}^{2} \cdot \mathrm{s} \cdot \mathrm{mmHg}\right)$ at $35^{\circ} \mathrm{C}$; * - gas method, ** — polarographic method); (3) hard $(n=1.42)$, semi-hard $(n=1.435)$, and soft $(n=1.39)$ contact lenses.

The specimens are exposed to following lasers: $\mathrm{CO}_{2}$, diode $(808 \mathrm{~nm})$, Er:YAG $(2.9 \mu \mathrm{m})$ and pulsed $\mathrm{Nd}^{3+}:$ YAG laser $(20 \mathrm{~mJ}, 15 \mathrm{~ns}, 1.064 \mu \mathrm{m})$. a

\section{Theory}

Laser induced damage threshold depends on material history before damaging and on its purity [1-3, 5-11]. LIDT differs for single-pulse and multi-pulse regimes. The modeling of damaging probability is complex and depends on both the laser type and working regime. Damage mechanisms are commonly treated by theories of fracture physics and mechanics, dielectric, Brillouin and thermal breakdowns, etc. $[1,10]$.

There are two theories treating the internal origins of damages. The first one does not make a difference between single- and multiple-pulse treatment. The internal damage threshold $T$ is a function of the number of pulses $N$ [5]. Another theory supposes that damages originated from different internal causes (origins) do not develop in the same way for the cases of single and multiple pulses. The damage threshold depends on the number of pulses logarithmically.

One of the modeling approaches [5] considers two phases of damaging. During the first one, under the influence of specific number of pulses, some variations inside the bulk appear; there are no visible damages on the surface. The other phase starts when internal modifications reach the critical level (one pulse damages surface). This is significant for the redundancy forecast of optical components in laser systems.

The electric field amplitude within the hollow made on uniform dielectric (with dielectric constant $\varepsilon$ ) is a function of incident electric field amplitude $E_{0}$ and is given by 


$$
E=[1+(1-\varepsilon) L / \varepsilon]^{-1} E_{0},
$$

where $L$ is depolarization factor depending on the shape of the hollow. Thus, for spherical hollow within the material $L=1 / 3$ and for cylindrical hollow on the surface $L=1 / 2$. For narrow, pin-like hollow $L=1-\pi c /(2 a)$, where $c$ is a diameter and $a$ is a hollow length. In the case of very narrow hollow $(c \ll a)$, $L=1$. The electric field amplitude becomes $E=\varepsilon E_{0}$, and the radiation intensity is $I=\varepsilon^{2} I_{0}$.

The laser radiation induces temperature change in a spherical region as a function of the distance from the sphere center $r$, and time $\tau$ is given by

$$
t(r, \tau)=t_{0} \exp \left(\tau \beta r^{2} / 3 a^{2}\right),
$$

where $t_{0}$ is the initial temperature, $\beta$ is the absorbance of the material, $a$ is the sphere radius.

The laser radiation intensity limit $I_{\mathrm{g}}$ can be expressed by

$$
I_{\mathrm{g}}=\frac{\lambda Q_{\mathrm{g}}}{185} \frac{\left(\varepsilon_{1}+2\right)^{2}+\varepsilon_{2}^{2}}{\varepsilon_{2}},
$$

where $Q_{\mathrm{g}}=C / a^{2}, \varepsilon_{1}$, and $\varepsilon_{2}$ are real and imaginary part of dielectric constant [10].

\section{Results and discussion}

For Ni sphere within a ruby and GaAs sphere, the temperature changes versus distance from the sphere center $r$ and time $\tau$ are obtained by applying Eq. (2) and presented in Fig. 1. In both cases the considered sphere radius was

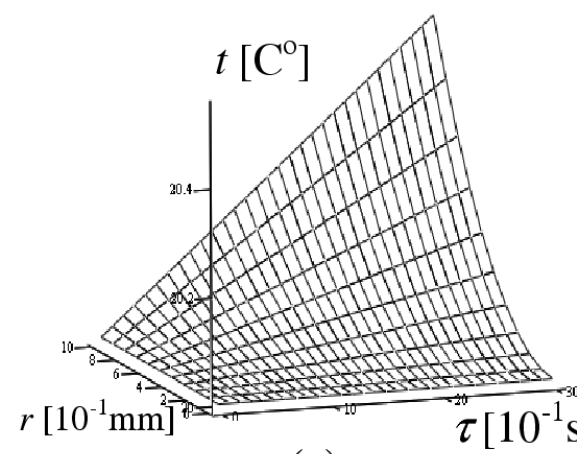

(a)

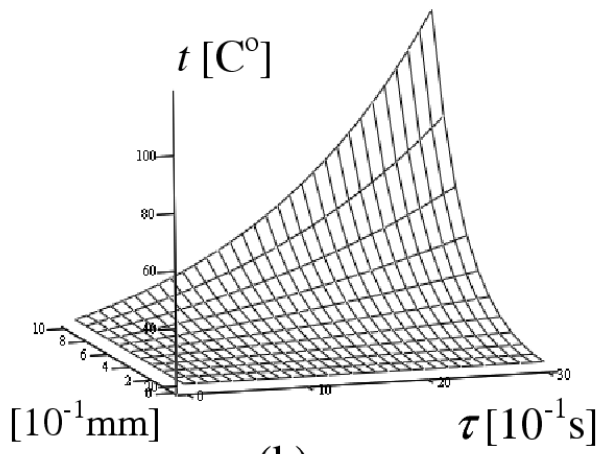

(b)

Fig. 1. The temperature changes versus distance from the sphere center $r$ and time $\tau$ for (a) GaAs sphere, and (b) Ni sphere within a ruby.

$a=1 \mathrm{~mm}$, and initial temperature was $t_{0}=20^{\circ} \mathrm{C}$. Due to small absorbance of GaAs $\left(\beta=2.7 \times 10^{-2} 1 / \mathrm{s}\right)$, the temperature in the sphere center remains the same and the temperature of the sphere surface rises up to only $20.55^{\circ} \mathrm{C}$ during the period of $3 \mathrm{~s}$. During the same period, in Ni sphere, with the absorbance of 

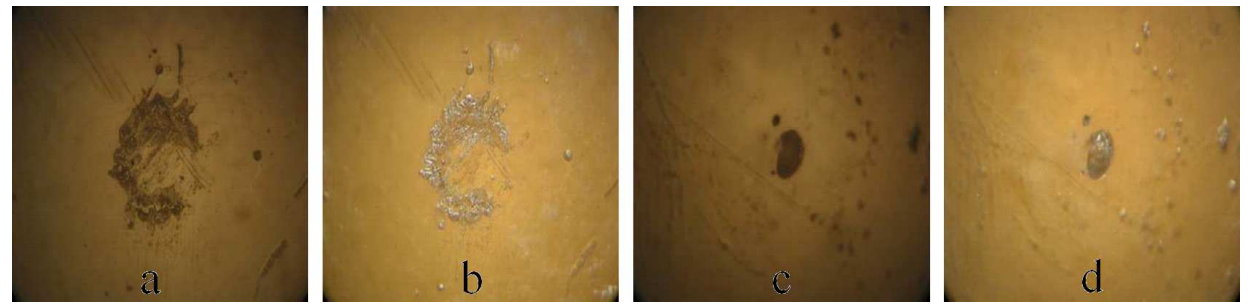

Fig. 2. Damages $(100 \times)$ on (a), (b) semi-hard and (c), (d) hard lens.

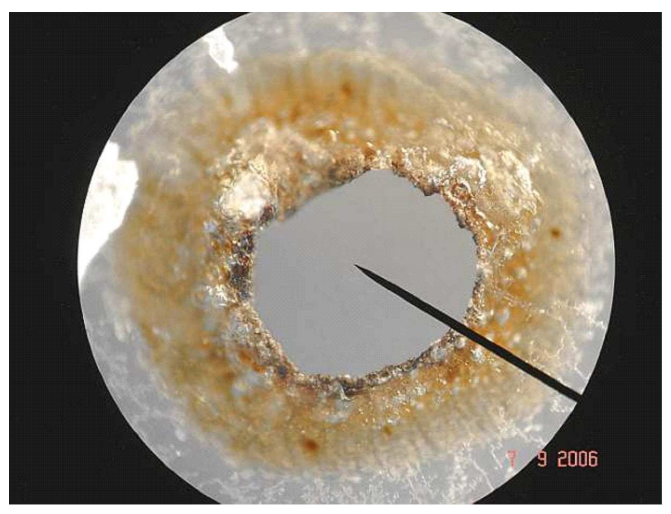

Fig. 3. Damages on lens of $20 \mathrm{~mm}$ diameter.

$1.791 / \mathrm{s}$, the temperature in the center rises slightly $\left(20.36^{\circ} \mathrm{C}\right)$ while the surface temperature rises up to $120^{\circ} \mathrm{C}$.

Damaged samples are analyzed by optical microscopy and fiber techniques. Some interesting damage cases (lenses) are presented in Fig. 2 and 3. Photorecording methods were applied for energy density calculations.

The resistance of optical components to laser beams is described by irradiation power density needed to produce the change in the component material, i.e. by damage threshold. The damage appears on the surface of the dielectric due to the ionization of absorbed contaminant molecules (mostly water). Multi-photon ionizations should be taken into account. For high quality dielectrics, this is the main source of damaging. Damages induced by thermally induced fractures due to energy absorption are rare.

Final mechanical processing of optical components is important in overall resistivity. Scratches and waves induced by bad polishing lead to reflection and diffraction effects as well as to the appearance of parasitic electric field and its changes associated with LIDT.

\section{Conclusion}

There are many models of the interaction between laser beams and glass/polymer-based optical components. Due to wide application area, there is a lot of 
empirical data concerning the interaction. Treated components are soft, semi-hard, and hard lenses, glasses for binoculars and other implementations, including for fine laser, holographic and interference optics. Damages provoked by $Q$-switched $\mathrm{Nd}$ :YAG are smaller compared to damages provoked by $\mathrm{CO}_{2}$ of significantly less power. There are several recognized procedures for the professional investigation of damage threshold. However, the thresholds are different even for the most known materials. Because stabilized lasers are rarely implemented in these experiments it would be interesting to conduct parallel research with this kind of lasers. In each laser experiment, the dosimetry is tightly connected to $\lambda, E, \tau, P$ and the pulse shape; for each estimate, the protocol should be respected $[1,11]$.

\section{Acknowledgments}

This work has been supported by the Ministry of Science, Republic of Serbia, under projects No. 141003.

\section{References}

[1] (a) Laser Induced Damage of Optical Materials, Ed. R.M. Wood, COSOLAS Ltd, London 2003; (b) R.M. Wood, Laser Damage in Optical Materials, Adam Hilger, Bristol 1986.

[2] M. Srećković, A. Marinović, V. Š ijački-Žeravić, S. Ristić, V. Krasnjuk, A. Milutinović-Nikolić, S. Pantelić, Ž. Tomić, in Proc. LASERS2001, Eds. V.J. Corcoran, T.A. Corcoran, STS Press, McLean (VA) 2002, p. 367.

[3] M. Sreković, N. Ivanović, V. Šijački-Žeravić, S. Ostojić, B. Vedlin, P. VujkovićCvijin, F. Borelić, R. Stepić, Opt. Las. Technol. 3, 169 (1991).

[4] O.V. Bogdankević, S.A. Darznek, P.G. Eliseev, Semiconductor Lasers, Nauka, Moskva 1976 (in Russian).

[5] M. Srećković, A. Marinović, A. Kovačević, D. Družijanić, S. Pantelić, N. Borna, G. Gligorić, D. Barjaktarević, S. Popović, in: Proc. XLIX ETRAN, Eds.M. Milošević, Z. Jakić, D. Boić, V. Potkonjak, Society for ETCAN, Beograd 2005, Book IV, p. 251.

[6] A. Kovačević, M. Srećković, R. Gospavić, S. Ristić, P. Jovanić, Acta Phys. Pol. A 112, 981 (2007).

[7] M. Srećković, A. Kovačević, M. Davidović, M. Dinulović, M. Kutin, A. Milosavljević, B. Dokić, in: AIP Conf. Proc. 876, of 23rd SSISPIG, Eds. Lj. Hadievskić, B.P. Marinković, N.S. Simonović, AIP, Melville (NY) 2006, p. 243.

[8] (a) J.F. Ready, Effects of High-Power Laser Radiation, Mir, Moscow 1974 (in Russian); (b) LIA Handbook of Laser Materials Processing, Eds. J. Ready, D. Farson, Magnolia, Orlando 2001; (c) N.N. Rykalin, A. Uglov, I. Zuev, A. Kokora, Laser and electron-beam material processing, Mir, Moscow 1988; russian original by Mashinostroenie, Moscow 1985.

[9] A. Kovačević, PhD Thesis, Fac. Electrical Engineering, Belgrade 2005.

[10] M. Srećković, P. Osmokrović, J. Ilić, S. Ostojić, R. Gospavić, S. Peić, Elektrotehnika 51, 9 (2002).

[11] D. Sliney, M. Wolbarsht, Safety with Lasers and Other Optical Sources, Plenum, New York 1980. 